OPEN ACCESS

Edited by:

Chien-Feng Li,

National Health Research Institutes,

Taiwan

Reviewed by:

Wanshan Li,

Chi Mei Medical Center, Taiwan

Ching-Chieh Yang,

Chi Mei Medical Center,

Taiwan

*Correspondence:

Zhenbo Hu

huzhenbo@wfmc.edu.cn

Zhen Cai

caiz@zju.edu.cn

${ }^{\dagger}$ These authors have contributed equally to this work

Specialty section:

This article was submitted to

Hematologic Malignancies,

a section of the journal

Frontiers in Oncology

Received: 15 January 2021 Accepted: 15 April 2021

Published: 17 May 2021

Citation:

Liu X, Li J, Wang Z, Meng J,

Wang A, Zhao X, XU Q, Cai Z and

Hu Z (2021) KDM2A Targets

PFKFB3 for Ubiquitylation to

Inhibit the Proliferation and

Angiogenesis of Multiple

Myeloma Cells.

Front. Oncol. 11:653788.

doi: 10.3389/fonc.2021.653788

\section{KDM2A Targets PFKFB3 for Ubiquitylation to Inhibit the Proliferation and Angiogenesis of Multiple Myeloma Cells}

\author{
Xinling Liu ${ }^{1 \dagger}$, Jiaqiu $L^{2+}{ }^{2}$, Zhanju Wang ${ }^{1}$, Jie Meng ${ }^{1}$, Aihong Wang ${ }^{1}$, Xiaofei Zhao ${ }^{3}$, Qilu \\ $\mathrm{Xu}^{4}$, Zhen $\mathrm{Cai}^{5 *}$ and Zhenbo $\mathrm{Hu}^{1 *}$ \\ ${ }^{1}$ Department of Hematology, Laboratory for Stem Cell and Regenerative Medicine, Clinical Research Center, Affiliated \\ Hospital of Weifang Medical University, Weifang, China, ${ }^{2}$ Department of Oncology, Clinical Research Center, Affiliated \\ Hospital of Weifang Medical University, Weifang, China, ${ }^{3}$ Department of Dermatology, Weifang Hospital of Traditional \\ Chinese Medicine, Weifang, China, ${ }^{4}$ Department of Hematology, The First Affiliated Hospital, Weifang Medical University, \\ Weifang, China, ${ }^{5}$ Department of Hematology, The First Affiliated Hospital, School of Medicine, Zhejiang University, \\ Hangzhou, China
}

The lysine demethylase KDM2A (also known as JHDM1A or FBXL11) demethylates histone $\mathrm{H} 3$ at lysine $\mathrm{K} 36$ which lead to epigenetic regulation of cell proliferation and tumorigenesis. However, many biological processes are mediated by KDM2A independently by its histone demethylation activity. In the present study, we aimed to characterize the functional significance of KDM2A in multiple myeloma (MM) disease progression. Specifically, we defined that one of the key enzymes of glycolysis PFKFB3 (6phosphofructo-2-kinase) is ubiquitylated by KDM2A which suppresses MM cell proliferation. Previous study showed that KDM2A and PFKFB3 promoted angiogenesis in various tumor cells. We further reveal that KDM2A targets PFKFB3 for ubiquitination and degradation to inhibit angiogenesis. Several angiogenic cytokines are also downregulated in MM. Clinically, MM patients with low KDM2A and high PFKFB3 levels have shown worse prognosis. These results reveal a novel function of KDM2A through ubiquitin ligase activity by targeting PFKFB3 to induce proliferation, glycolysis and angiogenesis in MM cells. The data provides a new potential mechanism and strategy for MM treatment.

Keywords: KDM2A, PFKFB3, ubiquitination, multiple myeloma, proliferation

\section{INTRODUCTION}

The pathogenic role of histone modification leads to various cancers (1). In the nucleosome, DNA is packaged by two sets of core histones, $\mathrm{H} 2 \mathrm{~A}, \mathrm{H} 2 \mathrm{~B}, \mathrm{H} 3$ and $\mathrm{H} 4$, whose amino acid residues can be regulated by diverse post-translational modifications such as methylation, acetylation or phosphorylation (2). The lysine demethylase KDM2A, also known as JHDM1A, FBXL11 or Ndy2, demethylates histone $\mathrm{H} 3$ at lysine K36 (3). Besides, KDM2A has been demonstrated to demethylate lysines of non-histone proteins such as the NF-kappaB, p65 subunit or beta-catenin $(4,5)$. KDM2A also 
stimulates ubiquitination and stabilizes p53-binding protein 1 (53BP1), promotes recruitment of 53BP1 to DNA breaks, and finally leads to increased DNA damage-induced genomic instability (6). Another lysine demethylase KDM2B, structurally similar to KDM2A, also exhibits an E3 ubiquitin ligase activity (7). Therefore, the range of KDM2A substrates might extend beyond histones. In addition, based on different context, KDM2A exhibits proliferative/antiproliferative properties in various cancers (8-11). However, the function of KDM2A in $\mathrm{MM}$ is rarely reported and understood.

Cancer cells prefer glycolysis as the major source of energy to maintain their proliferation. The 6-phosphofructo-2-kinase/fructose2,6-biphosphatase 3 (PFKFB3) plays a crucial role to boost glycolysis (12). Recently, PFKFB3 is demonstrated increasingly to be involved in non-glycolysis-dependent biological functions, such as angiogenesis (13), cell cycle regulation (14), and autophagy (15). PFKFB3 is frequently overexpressed in diverse cancers and stimulated by oncogenic signaling. As reported, PFKFB3 can be modified in various ways. For example, phosphorylation of PFKFB3 at Ser461 by AMPK increases glycolytic flux and promotes cancer cell proliferation (16). Carbon monoxide (CO) reduces methylation of PFKFB3, which causes metabolic rewiring toward the pentose phosphate pathway (PPP) and acquisition of chemoresistance. Ubiquitin-mediated proteasomal degradation of PFKFB3 protein also reroutes glycolysis to the PPP (17). Previously, we found PFKFB3 was overexpressed in MM (18). As thus, the rationale of this study is to understand if the role of $\mathrm{KDM} 2 \mathrm{~A}$ played in regulating proliferation of MM cells is through PFKFB3 ubiquitylation in order to learn underlining mechanism and develop new strategy for $\mathrm{MM}$ treatment. Here, we show that KDM2A induced ubiquitylation of glycolytic enzyme PFKFB3 and negatively regulated MM cell proliferation and angiogenesis.

\section{MATERIALS AND METHODS}

\section{Cell Culture and Reagents}

MM cell lines RPMI8226 and MM.1S were purchased from DSMZ (Braunschweig, Germany), and CAG and ARP1 were generously provided Dr. Qing Yi (Center for Hematologic Malignancy, Research Institute, Houston, TX, USA). All the cell lines were maintained in RPMI-1640 medium (Corning Cellgro, USA) supplemented with $10 \%$ fetal bovine serum (FBS) (GIBCO, CA, USA), $100 \mathrm{U} / \mathrm{ml}$ penicillin and $100 \mu \mathrm{g} / \mathrm{ml}$ streptomycin at $37^{\circ} \mathrm{C}$ and $5 \% \mathrm{CO}_{2}$ in air. $\mathrm{BM}$ samples from $\mathrm{MM}$ patients and peripheral blood mononuclear cells (PBMCs) from healthy donors were obtained after informed consent was provided following approval by the Ethics Committee of Affiliated Hospital of Weifang Medical University. MG132 and Cycloheximide were purchased from Sigma-Aldrich. Primary antibodies against KDM2A, PFKFB3, CDK6, MCL-1 and ubiquitin were purchased from Abcam (Cambridge, UK). Anti-VEGF antibody was purchased from Santa Cruz Biotechnology (Santa Cruz, CA, USA). GAPDH and Bax were purchased from Cell Signaling Technology (Danvers, USA). HA was obtained from Roche (Basel, Switzerland). Horseradish peroxidase (HRP)-conjugated anti-rabbit antibodies were obtained from Jackson ImmunoResearch Laboratories (Lancaster, USA). Lipofectamine 2000 reagent (Invitrogen) was used for transient transfection. The shRNA or siRNA sequences used were shown in Supplementary Table $\mathbf{S 1 .}$

\section{Cell Proliferation Assay}

A cell counting kit-8 (CCK-8) assay kit (Dojindo, Japan) was used to measure MM cell proliferation and viability. MM cells $\left(1 \times 10^{5} / 100 \mu \mathrm{l} /\right.$ well $)$ were seeded in 96 -well plates and treated with $10 \mu \mathrm{l}$ of CCK- 8 solution co-incubating at $37^{\circ} \mathrm{C}$ for $2 \mathrm{~h}$. Then, absorbance was measured at $450 \mathrm{~nm}$ using an enzyme microplate reader. Cell viability $(\%)=\mathrm{OD}$ value of the test sample/OD value of the control $\times 100$.

\section{Stable Cell Line Generation}

For stable KDM2A knockdown, MM cells were transfected with green fluorescent protein-containing (GFP) shRNA lentiviral particles directed against human KDM2A or with empty vector. Cells were selected in culture media containing puromycin $(2 \mu \mathrm{g} / \mathrm{ml})$ for 2 weeks.

\section{ELISA}

Human VEGF and IL-32 was determined by ELISA (R\&D Systems) according to the manufacturer's instructions.

\section{Glucose Uptake and Lactate Production Measurements}

The assays were carried out according to the protocol of glucose uptake assay kit (colorimetric, Abcam) and lactate Assay Kit (Cayman Chemical).

\section{Tube Formation Assays}

$2 \times 10^{5}$ cells were seeded on 24 -well plates coated with BD Matrigel $^{\mathrm{TM}}$ Matrix and incubated in indicated medium for $24 \mathrm{~h}$ at $37^{\circ} \mathrm{C}$. The formation of capillary-like structures was observed and captured under a light microscope. The total length of tubes was analyzed by ImageJ.

\section{Xenograft Tumor Studies}

CAG cells $\left(5 \times 10^{6}\right)$ with $\mathrm{KDM} 2 \mathrm{~A}$ overexpression resuspended in $50 \mu \mathrm{l}$ RPMI-1640 were injected subcutaneously into 4-week-old male NODSCID (non-obese diabetic-severe combined immunodeficient) mice. The experiments were terminated 3 weeks after cell injection, and tumors were weighed. Tumor volume was calculated according to the following formula: $\mathrm{V}=4 \pi / 3 \times(\mathrm{a} / 2)^{2} \times \mathrm{b} / 2$, where $\mathrm{a}$ is the tumor width and $b$ is the tumor length. The mice were sacrificed when the tumor volumes reached approximately $3000 \mathrm{~mm}^{3}$. All animal experiments were carried out in accordance with the protocols of the Animal Ethics Committee of the Affiliated Hospital of Weifang Medical University.

\section{Co-IP and Western Blot}

Whole-cell extracts were prepared in RIPA buffer (Cell Signaling Technology) with multiple protease inhibitors (Sigma-Aldrich) for $30 \mathrm{~min}$. Total protein was pre-cleared with Protein A/G beads which were incubated with rotation for $2 \mathrm{~h}$ at $4^{\circ} \mathrm{C}$. After centrifugation, the supernatant was moved to a new tube and the beads conjugated with specific antibody were added to the supernatant. The tube was rotated 
for $2 \mathrm{~h}$ at $4^{\circ} \mathrm{C}$ again. Then the tube was placed into a magnetic stand to collect the beads, and the supernatant was discarded. The beads were washed three times with wash buffer, and the complexes were eluted from the beads for sodium dodecyl sulfate-polyacrylamide gel electrophoresis (SDS-PAGE) and used for further analysis. Western blot was performed as described previously (18).

\section{Immunohistochemistry Analyses}

Paraformaldehyde-fixed, Triton X-100-permeabilized cells from BM biopsy tissues of MM patients were used for immunofluorescence staining with specific antibodies to analyze the expression of KDM2A and PFKFB3. The experiment was approved by the Affiliated Hospital of Weifang Medical University Ethics Committees. Immunostaining scores were independently evaluated by two researchers who were blinded to the patients and samples. The percent of positive staining cells $(<50 \%, 50-70 \%$, and $>70 \%)$ and the staining intensity were defined as following: absent $(-)$, scarce $( \pm)$, moderate $(+)$ or strong $(++)$. The expression levels of KDM2A and PFKFB3 were scored as follows: positive: $>70 \%$ positivity with scarce $( \pm)$ to strong $(++)$ staining or $50-70 \%$ positivity with moderate $(+)$ to strong $(++)$ staining; weakly positive: $50-70 \%$ positivity with scarce $( \pm)$ staining or $<50 \%$ positivity with scarce $( \pm)$ to strong $(++)$ staining; and negative: $<50 \%$ positivity with scarce $( \pm)$ to absent $(-)$ staining. The high expression group composed of positive cases, whereas the low expression group contained weakly positive and negative cases as defined above. The average sum of integrated optical density (IOD) of each sample was calculated using ImageJ software.

\section{Sample Preparation for MS and LC- ESIMS/MS Analysis by $Q$ Exactive HF}

The purified protein samples prepared from IP were separated by SDS-PAGE and subjected to LC-ESIMS/MS analysis which was performed by Micrometer Biotech Company (Hangzhou, China).

\section{Statistical Analysis}

All data were presented as the mean \pm standard deviation (S.D.). All analyses were conducted using GraphPad Prism 6.0 (GraphPad Software, San Diego, CA, USA). Two-tailed Student's t-test was used to estimate significant differences between two groups. $\mathrm{P}^{*}<0.05$ was considered to be significant. All experiments were performed in triplicate as three independent assays.

\section{RESULTS}

\section{PFKFB3 Is a Substrate of KDM2A}

Our previous study has demonstrated that metabolic enzyme PFKFB3 promoted MM cell proliferation. By mass spectrometry analysis, we demonstrated that PFKFB3 may be ubiquitylated and identified $\mathrm{KDM} 2 \mathrm{~A}$ as a new binding target. The representative spectra of KDM2A and PFKFB3 are shown in Supplementary Figure 1A. Firstly, the expression of KDM2A and PFKFB3 was detected by western blotting in MM cell lines, PBMCs and 293T cells. PFKFB3 protein showed obviously high expression in MM cells except of relatively low expression in MM.1S. KDM2A protein had relatively low expression level (Figure 1A). To further explore the underlying mechanism of PFKFB3 oncogenic function, the
A

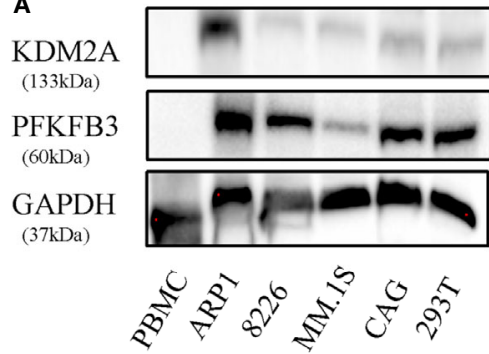

B

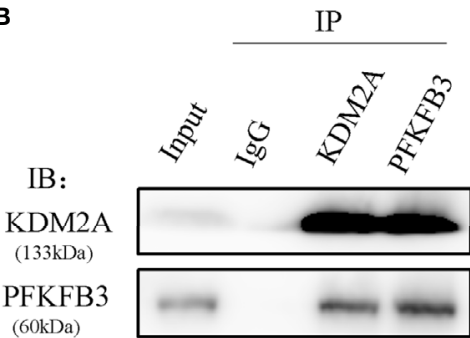

C

RPMI8226

KDM2A

(133kDa)

PFKFB3

(60kDa)

GAPDH

(37kDa)

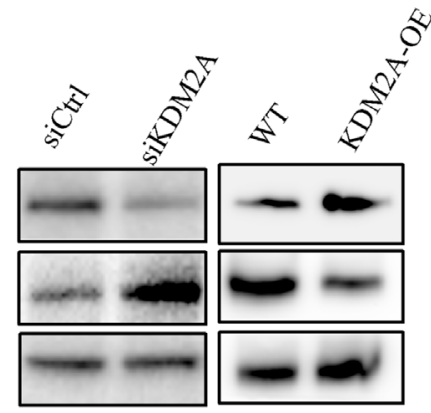

CAG

KDM2A

(133kDa)

PFKFB3

(60kDa)

GAPDH

(37kDa)

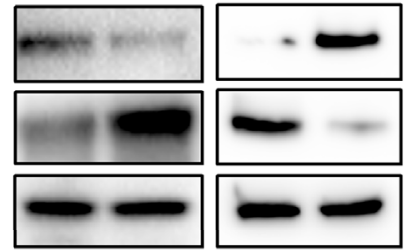

FIGURE 1 | PFKFB3 is a substrate of KDM2A. (A) Westem blot analysis of expression of PFKFB3 and KDM2A in MM cell lines, PBMCs and 293T cells. PBMC: normal healthy donors' peripheral blood mononuclear cells as the control. (B) Co-IP assay to detect that endogenous KDM2A and PFKFB3 interacted with each other in RPMl8226 cells. (C) Western blot analysis showed that PFKFB3 protein levels increased in RPMI8226 and CAG cells transfected with siKDM2A. The gain-of-function assays validated the suppressive effect of KDM2A on PFKFB3 as well. GAPDH was used as a loading control. All the experiments were repeated three times. 
interaction between PFKFB3 and KDM2A was determined by coimmunoprecipitation (Co-IP) assay in RPMI8226 cells (Figure 1B). Furthermore, Downregulation of KDM2A gene expression using small interfering RNA (siRNA) increased PFKFB3 protein expression level in RPMI8226 and CAG cell lines. Therefore, the gain-of-function assays validated the suppressive effect of KDM2A on PFKFB3 in MM cells (Figure 1C). Taken together, our results provide evidence that PFKFB3 is a direct target of KDM2A.

\section{KDM2A Promotes MM Cell Proliferation and Cell Cycle Progression}

To learn if the biological functions of KDM2A and PFKFB3 in MM cells are opposite, we measured the effect of KDM2A on
MM cell proliferation in vitro. The results showed that knockdown of KDM2A using small hairpin RNA (shRNA) promoted cell proliferation (Figure 2A). Due to promoting cell cycle progression as a non-canonical function of PFKFB3, we observed that in MM cells shKDM2A increased CDK6 protein level, which was involved in the core cell cycle that drove cell proliferation $(19,20)$. Moreover, myeloid cell leukemia-1 (MCL1) as an anti-apoptotic protein was increased and Bax as proapoptotic protein was decreased after KDM2A knockdown (Figure 2B). Consistently, overexpression of KDM2A inhibited myeloma tumor growth in vivo (Figures 2C, D). Collectively, these data suggest that $s h K D M 2 A$ increases $M M$ cell proliferation.
A
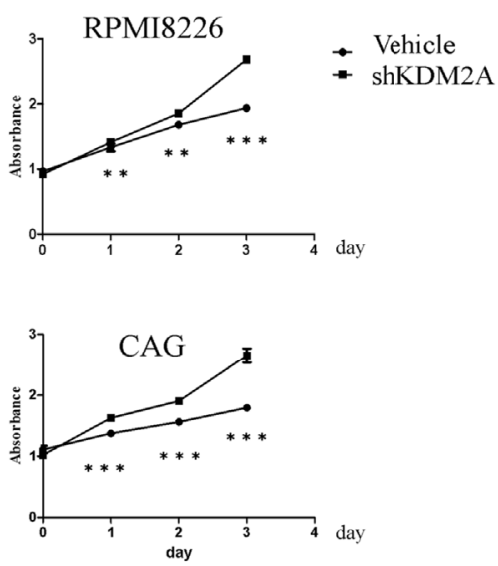

C

WT

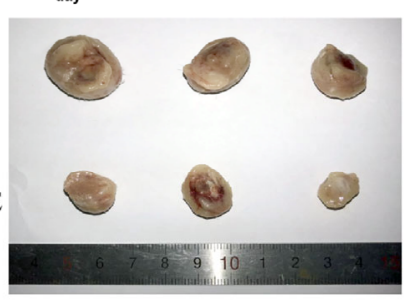

B

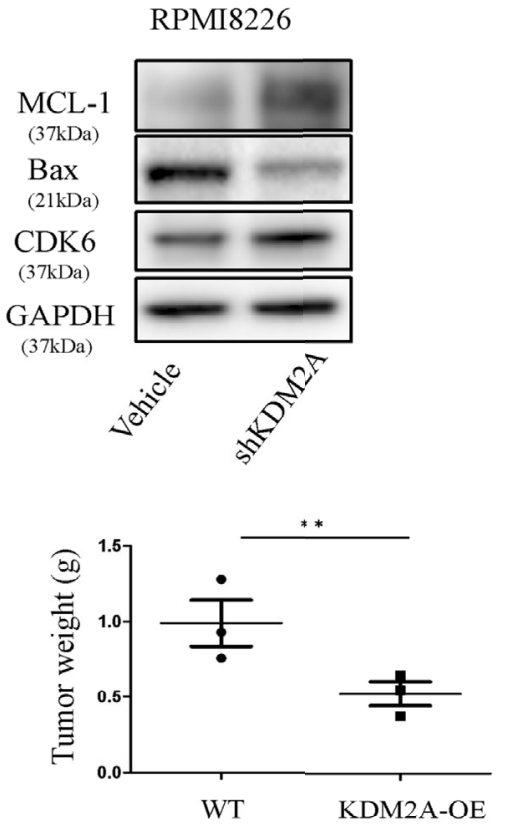

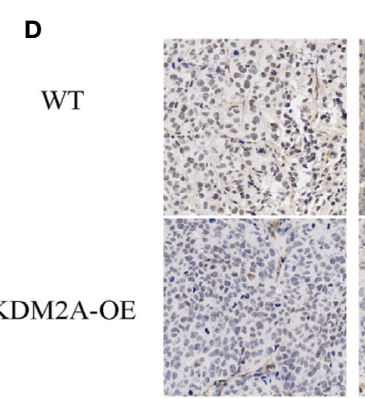

PFKFB3

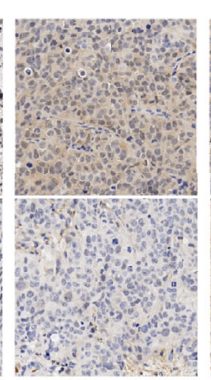

MCL-1

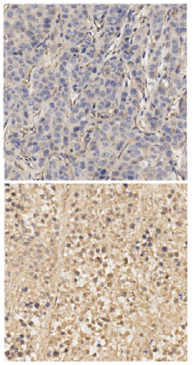

Bax

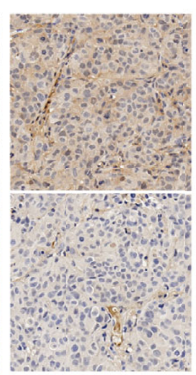

CDK6

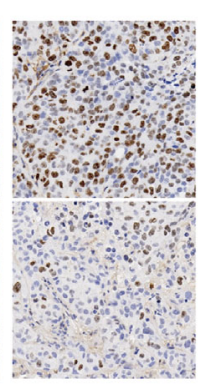

Ki67

FIGURE 2 | KDM2A mediates MM cell proliferation. (A) Proliferation of RPMI8226 and CAG cells was determined by CCK-8 assay after shKDM2A transfection. The results are representative of three independent experiments. (B) Knocking down of KDM2A decreased the expression of Bax and increased MCL-1 and CDK6 in MM cells by Western blot analysis. GAPDH was used as loading control. (C) Photographs of tumor masses were shown on the left panel and the tumor weight was measured and shown on the right panel after excised 3 weeks inoculation of CAG cells with KDM2A-WT or KDM2A-OE into NOD-SCID mice. (D) Immunohistochemistry staining for tumor tissue from KDM2A-WT and KDM2A-OE NOD-SCID Mice. ( ${ }^{\star} P<0.05$, ${ }^{\star \star} P<0.01$, ${ }^{\star \star \star} P<0.005$, data were represented as mean $\pm \mathrm{SD}$ ). 


\section{shKDM2A Promotes Glycolysis Activity and Tube Formation of HUVECs}

AMP-activated protein kinase (AMPK) maintains energy homeostasis, which can positively regulate PFKFB3 to promote glycolysis, thereby increasing glucose uptake for cancer cell proliferation (21). A study reported that inhibiting mTOR finally reduced PFKFB3 expression to negatively regulate angiogenesis and cell migration (22). It has been widely confirmed that PFKFB3 mediated tumor growth and angiogenesis $(23,24)$. In order to detect whether shKDM2A plays a similar role in glycolysis as that of PFKFB3, we determined the levels of glucose uptake and lactate production in MM cells. As shown in Figure 3A, glucose uptake and lactate production were both increased in MM cells, which means glycolysis enhanced with KDM2A knockdown. In addition, vascular endothelial growth factor (VEGF) is a key component of pro-angiogenic activity, and our previous study also demonstrated that IL-32 promoted angiogenesis. In consideration of high expression of IL-32 in multiple myeloma $(25,26)$, we then measured the levels of VEGF and IL-32 in MM cells by ELISA. As expected, the median levels of VEGF and IL32 were higher in the shKDM2A group than in the control group (Figure 3B). Meanwhile, immunohistochemical analysis showed that $\mathrm{KDM} 2 \mathrm{~A}-\mathrm{OE}$ tumors exhibited more negative staining for VEGF than control group (Figure 3C). Based on above data, tube formation assay further revealed that KDM2A knockdown promoted the formation of capillary-like structures in HUVECs (Figures 3D, E). These data indicate that shKDM2A promotes glycolysis activity and tube formation of HUVECs.

\section{KDM2A Induces Instability of PFKFB3}

As shown in Figure 1C, KDM2A negatively regulated endogenous PFKFB3 protein level. We next examined whether KDM2A regulates the stability of PFKFB3 protein. Firstly, we performed Co-IP assay in HEK293T to detect the exogenous interaction of KDM2A and PFKFB3 (Figure 4A). Then we overexpressed Flagtagged KDM2A in 293T cells which showed dramatically decrease PFKFB3 protein level in a dose-dependent manner (Figure 4B). Treatment of cells with MG132, a 26S proteasome inhibitor, effectively blocked PFKFB3 degradation (Figure 4C), confirming that PFKFB3 is degraded by KDM2A through the proteasome pathway. Furthermore, we treated cells with cycloheximide (CHX) to measure the half-life of PFKFB3. The knockdown of KDM2A decreased the degradation of PFKFB3 compared with the control vector (Figures 4D-F). Meanwhile, to determine whether the proteasome pathway is necessary for regulating of PFKFB3 protein stability, we treated the cells with $\mathrm{CHX}$ or MG132 for 120min and determined the PFKFB3 protein level by Western blotting. The result showed that MG132 inhibited the degradation of PFKFB3 protein (Figure 4G) suggesting proteasome pathway was involved in PFKFB3 protein degradation.

\section{Interaction Between KDM2A and PFKFB3}

The above Co-IP assay results showed that endogenous or exogenous KDM2A and PFKFB3 were bounded each other. Furthermore, we established PFKFB3 mutant with deletion of VLVIC 383-387, which is a potential SUMO-interacting motifs. The process of SUMOylation is putting a small ubiquitinlike
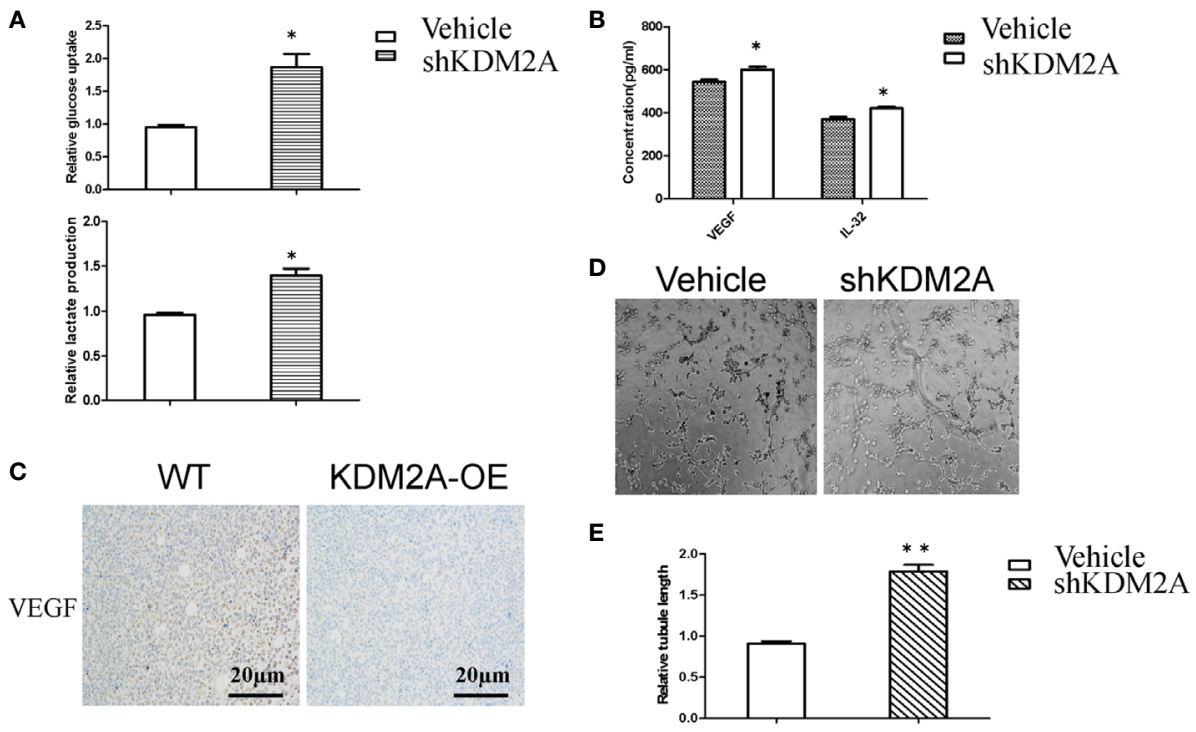

FIGURE 3 | shKDM2A promotes glycolysis activity and tube formation of HUVECs. (A) Glucose uptake and lactate production were detected in MM cells with shKDM2A as described in the Material and Methods using a glucose uptake assay kit and a lactate assay Kit. ${ }^{*} \mathrm{P}<0.05$. (B) ELISA assays showed that shKDM2A MM cells increased secretion of VEGF and IL-32 in culture medium. ${ }^{*} \mathrm{P}<0.05$. (C) Representative IHC images for VEGF in the mice were shown (scale bar, $20 \mu \mathrm{m}$ ). (D) MM cells with shKDM2A culture medium enhanced tube formation ability in HUVECs seeded on Matrigel. Images were taken using a light microscope ( $\times 100)$. (E) The quantitative data of the tube formation assays. Quantitative data were from 3 independent experiments. ${ }^{*} \mathrm{P}<0.05$. The above representative results were verified in RPMI8226 cell lines. 


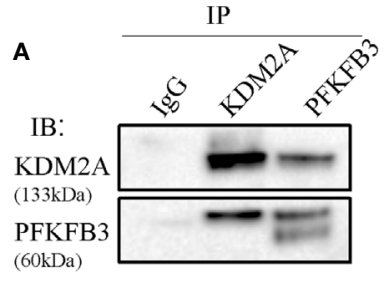

C

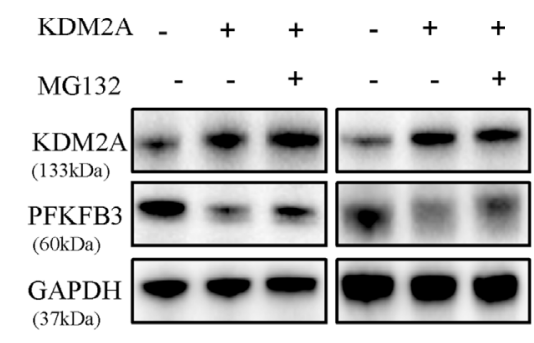

E

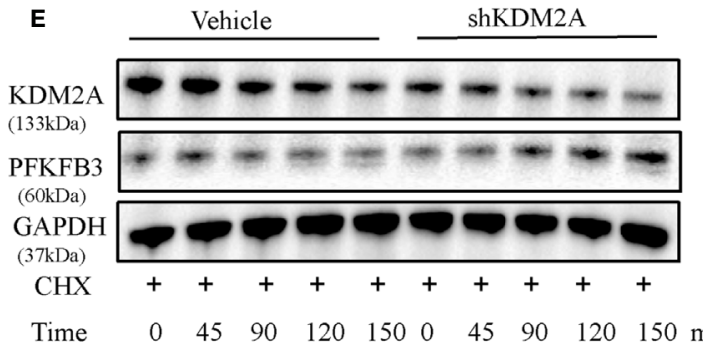

B Flag-KDM2A $0 \quad 1 \quad 2 \mu \mathrm{g}$

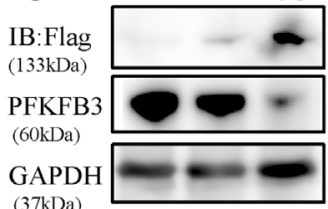

D

$$
\text { Time } 0 \quad 45 \quad 90 \quad 120 \mathrm{~min}
$$

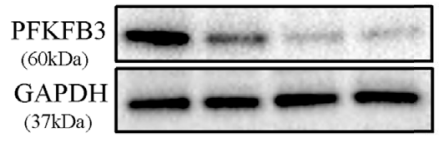

(37kDa)
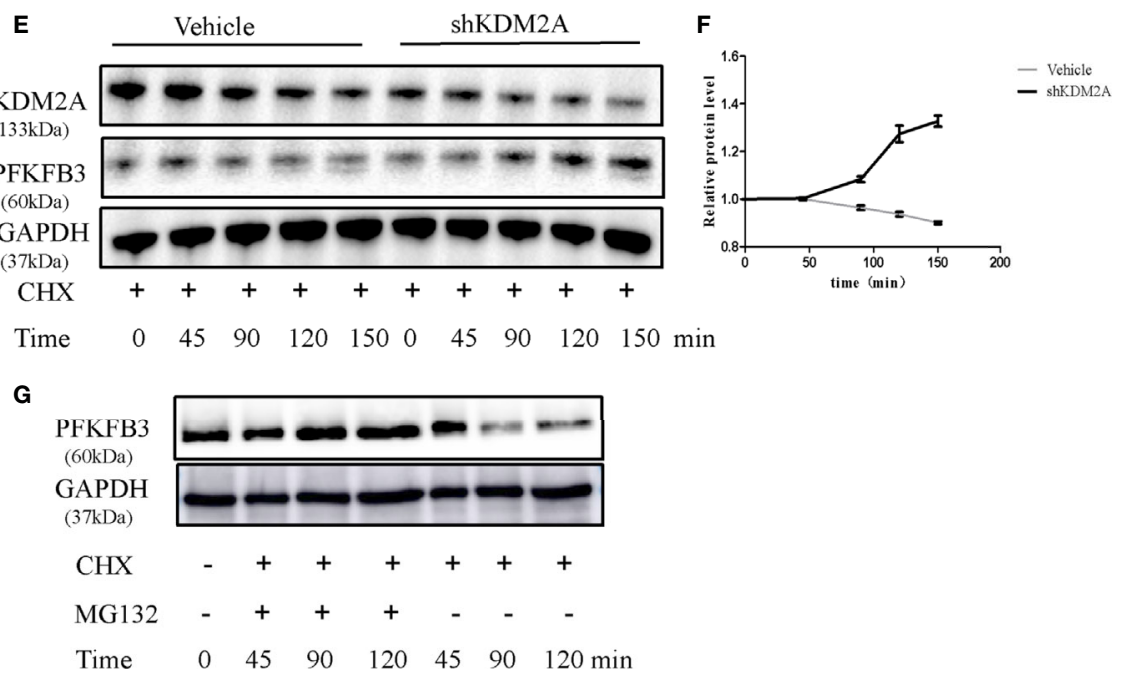

FIGURE 4 | KDM2A induces instability of PFKFB3. (A) Endogenous KDM2A and PFKFB3 interacted with each other in 293T cells, which was determined by Co-IP assay and Western blot. (B) Dose-dependent reduction of PFKFB3 in 293T cells transfected with KDM2A expression vector. GAPDH was used as loading control. (C) KDM2A induced ubiquitin proteasome system of PFKFB3. MM cells with KDM2A overexpression were treated with MG132 for 2 hours. PFKFB3 protein expression was evaluated as determined by Western blotting. (D) PFKFB3 protein degradation in MM cells treated with CHX in a time series. (E) Comparison of PFKFB3 protein degradation between the wild-type KDM2A and shKDM2A in cells with a time-course CHX treatment. (F) Quantification of (E) in triplicate.

(G) MG132 inhibited the degradation of PFKFB3 protein detected by western blot analysis.

modifier (SUMO) to protein substrates at specific lysine site (27, 28). Then we observed that KDM2A did not induce the degradation of PFKFB3 mutant (Figure 5A). Consistently, KDM2A overexpression did not change the stability of PFKFB3 mutant (Figure 5B), suggesting that the latter was resistant to KDM2A. As shown in Figure 5C, KDM2A strongly enhanced the ubiquitylation of PFKFB3. Moreover, KDM2A induced a dramatic enhancement in the ubiquitylation of PFKFB3, but not of the PFKFB3 mutant (Figure 5D). In summary, KDM2A interacts specifically with PFKFB3 to induce its degradation by ubiquitination pathway.

\section{Protein Levels of KDM2A and PFKFB3 Are Negatively Correlated in Multiple Myeloma Patients}

To further analyze the relationship between KDM2A and PFKFB3, bone marrow (BM) samples $(n=14)$ from MM patients were analyzed by immunohistochemistry. Statistical analysis showed that with the severity of the disease, KDM2A protein levels decreased (Figures 6A-C). Meanwhile, BM tissue with high expression of KDM2A exhibited a low expression of PFKFB3 protein, and vice versa. In order to ascertain the correlation of KDM2A and PFKFB3 expression, Pearson 
A

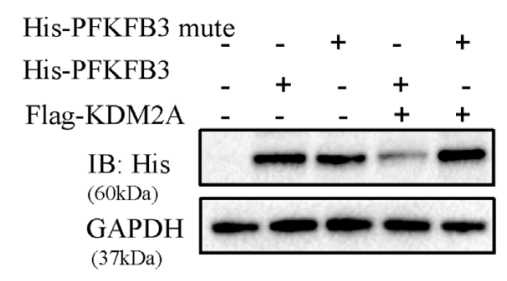

B His-PFKFB3 mute

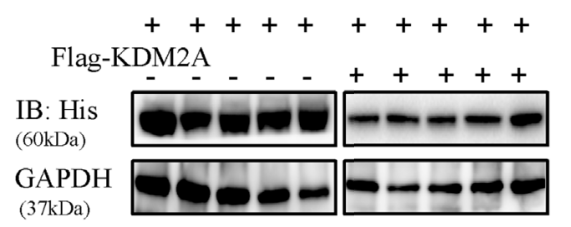

CHX $\quad 0 \quad 45 \quad 90120150 \quad 0 \quad 45 \quad 90 \quad 120150 \mathrm{~min}$

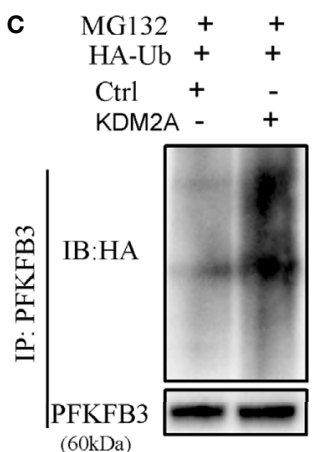

D

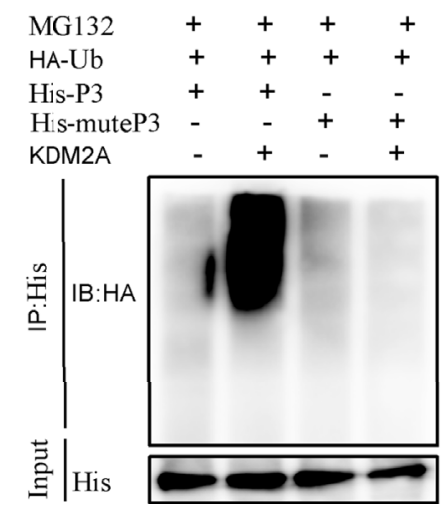

FIGURE 5 | Determination of interaction between KDM2A and PFKFB3. (A) Detection of the effect of KDM2A on PFKFB3 or PFKFB3 mutant degradation in 2937 cells. (B) The turnover rate of PFKFB3 mutant was not changed in response to KDM2A overexpression with CHX treatment. (C) KDM2A generated ubiquitylation on PFKFB3. (D) KDM2A overexpression induced strong ubiquitylation of PFKFB3, but not on the PFKFB3 mutant protein.

A

\section{KDM2A}

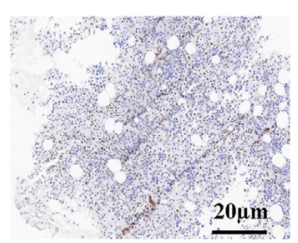

$20 \mu \mathrm{m}$
PFKFB3

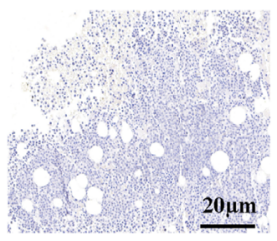

C

KDM2A

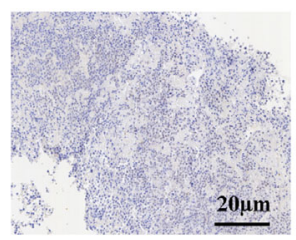

PFKFB3

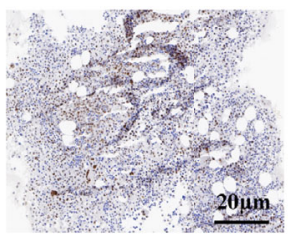

B

KDM2A

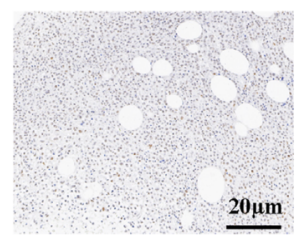

D

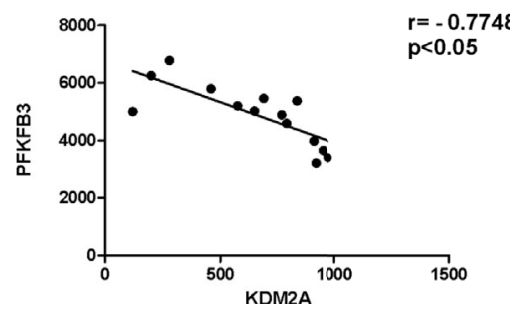

FIGURE 6 | Protein levels of KDM2A and PFKFB3 are negatively correlated in multiple myeloma patients. (A-C) Representative images of staining with KDM2A antibodies in MM patients' BM tissues showed strong (A), moderate (B), and weak (C) expression, respectively, and the expression of PFKFB3 is just the opposite of KDM2A (scale bar, 20 um). (D) Pearson correlative analysis of semi-quantitative IOD values for KDM2A and PFKFB3 indicates that protein levels of KDM2A and PFKFB3 are negatively correlated in MM patients. 
TABLE 1 | Analysis of correlation between KDM2A or PFKFB3 protein levels and clinicopathological parameters of multiple myeloma patients.

\begin{tabular}{|c|c|c|c|c|c|c|c|c|}
\hline \multirow[b]{2}{*}{ Variable } & \multicolumn{4}{|c|}{ KDM2A expression } & \multicolumn{4}{|c|}{ PFKFB3 expression } \\
\hline & $\mathbf{n}$ & Low & High & p-Value & $\mathbf{n}$ & Low & High & p-Value \\
\hline & & & & 0.481 & & & & 0.052 \\
\hline \multicolumn{9}{|l|}{ Age } \\
\hline$\leq 60$ years & 10 & 7 & 3 & & 10 & 2 & 8 & \\
\hline$<60$ years & 4 & 2 & 2 & & 4 & 3 & 1 & \\
\hline Gender & & & & 0.027 & & & & 0.207 \\
\hline Female & 11 & 8 & 3 & & 11 & 3 & 1 & \\
\hline ISS & & & & 0.027 & & & & 0.030 \\
\hline Stage I & 5 & 1 & 4 & & 5 & 4 & 1 & \\
\hline Stage II & 4 & 3 & 1 & & 5 & 4 & 1 & \\
\hline Stage III & 5 & 5 & 0 & & 5 & 1 & 4 & \\
\hline Durie-Salmon staging & & & & 0.543 & & & & 0.543 \\
\hline Stage IIA & 1 & 1 & 0 & & 1 & 0 & 1 & \\
\hline Stage IIB & 2 & 2 & 0 & & 2 & 1 & 1 & \\
\hline Stage IIIA & 9 & 5 & 4 & & 9 & 4 & 5 & \\
\hline Stage IIIB & 2 & 1 & 1 & & 2 & 0 & 2 & \\
\hline
\end{tabular}

correlation test was performed based on IOD values. The Pearson correlation coefficient of KDM2A and PFKFB3 was -0.77 (Figure 6D), indicating that the expression of the two proteins was negatively correlated. Consistent with these data, we found that the KDM2A and PFKFB3 protein levels were significantly correlated with the ISS (International Staging System) stage of multiple myeloma, but not with other clinicopathological parameters (Table 1), which indicated that KDM2A and PFKFB3 may play a role in MM progression.

\section{DISCUSSION}

Studies have shown that KDM2A as a lysine demethylase was highly overexpressed in many cancers, such as breast cancer $(8$, 29), gastric cancer (9), lung cancer (30) and hepatoma (31). In addition, it can be downregulated by tumor microenviroment (28). In the present study, we demonstrated that expression of KDM2A was basically low in multiple myeloma cells. KDM2A mainly demethylates histone $\mathrm{H} 3 \mathrm{~K} 36$, however, more and more studies demonstrate that KDM2A has non-histone targets $(4,5$, 32). Our data demonstrates that in myeloma cells KDM2A interacts with PFKFB3 and mediates its ubiquitination-induced degradation. Moreover, KDM2A knockdown promotes MM tumorigenesis and progression.

PFKFB3 is one of the pivotal glycolytic enzymes which promotes cancer cell proliferation (33-35). Our previous studies indicate that PFKFB3 inhibition alleviated MM cell proliferation (18). In addition, the function of PFKFB3 is regulated by protein stability and transcriptional stimulation. For example, hypoxia stimulated PFKFB3 expression at the transcriptional level (36). PFKFB3 is subjected to proteosomal degradation through the E3 ubiquitin ligase APC/C-Cdh1 (37). LncRNA AGPG stabilizes PFKFB3 by preventing K302 ubiquitination (35). PFKFB3 activity is also regulated by posttranslational modifications, such as phosphorylation (38), dimethylation (17) and acetylation (39). This study is the first to report PFKFB3 can be ubiquitylated by KDM2A in multiple myeloma. Meanwhile, previous studies reported that tumor angiogenesis can be promoted by PFKFB3-mediated glycolysis $(24,40)$, and the production of VEGF, the most powerful proangiogenic growth factor, is increased. Moreover, IL-32 as a proinflammatory factor has been demonstrated to promote angiogenesis (41). Our previous study showed that IL-32 was overexpressed in MM patients (42). In this study, we demonstrate that knockdown of KDM2A increases PFKFB3 level, which further increases IL-32 mRNA to enhance tube formation in HUVECs. Therefore, our finding is consistent with the other studies.

In summary, the present study demonstrates that the ubiquitination of PFKFB3 by KDM2A inhibits MM tumorigenesis. In other words, a decreased level of KDM2A in MM patients may indicate a poor clinical prognosis. Our findings further suggest that regulating KDM2A-mediated PFKFB3 ubiquitination may be a promising application in multiple myeloma treatment.

\section{DATA AVAILABILITY STATEMENT}

The raw data supporting the conclusions of this article will be made available by the authors, without undue reservation.

\section{ETHICS STATEMENT}

The studies involving human participants were reviewed and approved by Affiliated Hospital of Weifang Medical University. The patients/participants provided their written informed consent to participate in this study. The animal study was reviewed and approved by Affiliated Hospital of Weifang Medical University.

\section{AUTHOR CONTRIBUTIONS}

$\mathrm{XL}$ designed and performed the experiments. JL performed experiments. ZW, JM, AW and XZ helped in experiments and 
analyzed data. ZW and QX collected primary samples for the study. XL, ZC and ZH supervised the study, wrote and revised the paper. All authors contributed to the article and approved the submitted version.

\section{FUNDING}

This study was supported by Projects of medical and health technology development program in Shandong province (Grant No. 2019WS601, 2013WS0291), Shandong Province

\section{REFERENCES}

1. Dawson MA, Kouzarides T. Cancer Epigenetics: From Mechanism to Therapy. Cell (2012) 150(1):12-27. doi: 10.1016/j.cell.2012.06.013

2. Bannister AJ, Kouzarides T. Regulation of Chromatin by Histone Modifications. Cell Res (2011) 21(3):381-95. doi: 10.1038/cr.2011.22

3. Tsukada Y, Fang J, Erdjument-Bromage H, Warren ME, Borchers CH, Tempst P, et al. Histone Demethylation by a Family of JmjC Domain-Containing Proteins. Nature (2006) 439(7078):811-6. doi: 10.1038/nature04433

4. Lu L, Gao Y, Zhang Z, Cao Q, Zhang X, Zou J, et al. Kdm2a/B Lysine Demethylases Regulate Canonical Wnt Signaling by Modulating the Stability of Nuclear Beta-Catenin. Dev Cell (2015) 33(6):660-74. doi: 10.1016/ j.devcel.2015.04.006

5. Lu T, Jackson MW, Wang B, Yang M, Chance MR, Miyagi M, et al. Regulation of NF-kappaB by NSD1/FBXL11-dependent Reversible Lysine Methylation of P65. Proc Natl Acad Sci USA (2010) 107(1):46-51. doi: 10.1073/pnas.0912493107

6. Bueno MTD, Baldascini M, Richard S, Lowndes NF. Recruitment of Lysine Demethylase 2A to DNA Double Strand Breaks and its Interaction With 53BP1 Ensures Genome Stability. Oncotarget (2018) 9(22):15915-30. doi: 10.18632/oncotarget.24636

7. Janzer A, Stamm K, Becker A, Zimmer A, Buettner R, Kirfel J. The H3K4me3 Histone Demethylase Fbxl10 is a Regulator of Chemokine Expression, Cellular Morphology, and the Metabolome of Fibroblasts. J Biol Chem (2012) 287(37):30984-92. doi: 10.1074/jbc.M112.341040

8. Liu H, Liu L, Holowatyj A, Jiang Y, Yang ZQ. Integrated Genomic and Functional Analyses of Histone Demethylases Identify Oncogenic KDM2A Isoform in Breast Cancer. Mol Carcinogenesis (2016) 55(5):977-90. doi: $10.1002 / \mathrm{mc} .22341$

9. Huang Y, Liu Y, Yu L, Chen J, Hou J, Cui L, et al. Histone Demethylase KDM2A Promotes Tumor Cell Growth and Migration in Gastric Cancer. Tumour Biology: J Int Soc Oncodevelopmental Biol Med (2015) 36(1):271-8. doi: 10.1007/s13277-014-2630-5

10. Batie M, Druker J, D'Ignazio L, Rocha S. KDM2 Family Members are Regulated by HIF-1 in Hypoxia. Cells (2017) 6(1):8. doi: 10.3390/cells6010008

11. Tanaka Y, Okamoto K, Teye K, Umata T, Yamagiwa N, Suto Y, et al. Jmjc Enzyme KDM2A is a Regulator of rRNA Transcription in Response to Starvation. EMBO J (2010) 29(9):1510-22. doi: 10.1038/emboj.2010.56

12. Cavalier MC, Kim SG, Neau D, Lee YH. Molecular Basis of the fructose-2,6bisphosphatase Reaction of PFKFB3: Transition State and the C-terminal Function. Proteins (2012) 80(4):1143-53. doi: 10.1002/prot.24015

13. Yang JG, Wang WM, Xia HF, Yu ZL, Li HM, Ren JG, et al. LymphotoxinAlpha Promotes Tumor Angiogenesis in HNSCC by Modulating Glycolysis in a PFKFB3-dependent Manner. Int J Cancer (2019) 145(5):1358-70. doi: $10.1002 /$ ijc. 32221

14. Yalcin A, Clem BF, Imbert-Fernandez Y, Ozcan SC, Peker S, O'Neal J, et al. 6Phosphofructo-2-kinase (PFKFB3) Promotes Cell Cycle Progression and Suppresses Apoptosis Via Cdk1-mediated Phosphorylation of P27. Cell Death Dis (2014) 5:e1337. doi: 10.1038/cddis.2014.292

15. Yan S, Wei X, Xu S, Sun H, Wang W, Liu L, et al. 6-Phosphofructo-2-kinase/ fructose-2,6-bisphosphatase Isoform 3 Spatially Mediates Autophagy Through the AMPK Signaling Pathway. Oncotarget (2017) 8(46):80909-22. doi: 10.18632/oncotarget.20757
Natural Science Foundation (Grants No. ZR2020QH096, ZR2020KC016), National Natural Science Foundation of China (Grant No. 81570157), Weifang Science and Technology Bureau (Grant No. 2020YQFK013).

\section{SUPPLEMENTARY MATERIAL}

The Supplementary Material for this article can be found online at: https://www.frontiersin.org/articles/10.3389/fonc.2021.653788/ full\#supplementary-material

16. Bando H, Atsumi T, Nishio T, Niwa H, Mishima S, Shimizu C, et al. Phosphorylation of the 6-phosphofructo-2-kinase/fructose 2,6Bisphosphatase/PFKFB3 Family of Glycolytic Regulators in Human Cancer. Clin Cancer Res: an Off J Am Assoc Cancer Res (2005) 11(16):5784-92. doi: 10.1158/1078-0432.CCR-05-0149

17. Yamamoto T, Takano N, Ishiwata K, Ohmura M, Nagahata $Y$, Matsuura T, et al. Reduced Methylation of PFKFB3 in Cancer Cells Shunts Glucose Towards the Pentose Phosphate Pathway. Nat Commun (2014) 5:3480. doi: 10.1038/ncomms4480

18. Liu X, Zhao Y, Zhang E, Yan H, Lv N, Cai Z. The Synergistic Effect of PFK15 With Metformin Exerts Anti-Myeloma Activity Via PFKFB3. Biochem Biophys Res Commun (2019) 515(2):332-8. doi: 10.1016/j.bbrc.2019.05.136

19. Jia W, Zhao X, Zhao L, Yan H, Li J, Yang H, et al. Non-Canonical Roles of PFKFB3 in Regulation of Cell Cycle Through Binding to CDK4. Oncogene (2018) 37(13):1685-98. doi: 10.1038/s41388-017-0072-4

20. Sherr CJ, Beach D, Shapiro GI. Targeting CDK4 and CDK6: From Discovery to Therapy. Cancer Discovery (2016) 6(4):353-67. doi: 10.1158/21598290.CD-15-0894

21. Domenech E, Maestre C, Esteban-Martinez L, Partida D, Pascual R, Fernandez-Miranda G, et al. AMPK and PFKFB3 Mediate Glycolysis and Survival in Response to Mitophagy During Mitotic Arrest. Nat Cell Biol (2015) 17(10):1304-16. doi: 10.1038/ncb3231

22. Ziegler ME, Hatch MM, Wu N, Muawad SA, Hughes CC. mTORC2 Mediates CXCL12-induced Angiogenesis. Angiogenesis (2016) 19(3):359-71. doi: 10.1007/s10456-016-9509-6

23. Cruys B, Wong BW, Kuchnio A, Verdegem D, Cantelmo AR, Conradi LC, et al. Glycolytic Regulation of Cell Rearrangement in Angiogenesis. Nat Commun (2016) 7:12240. doi: 10.1038/ncomms12240

24. Bousseau S, Vergori L, Soleti R, Lenaers G, Martinez MC, Andriantsitohaina R. Glycosylation as New Pharmacological Strategies for Diseases Associated With Excessive Angiogenesis. Pharmacol Ther (2018) 191:92-122. doi: 10.1016/j.pharmthera.2018.06.003

25. Zahoor M, Westhrin M, Aass KR, Moen SH, Misund K, Psonka-Antonczyk KM, et al. Hypoxia Promotes IL-32 Expression in Myeloma Cells, and High Expression is Associated With Poor Survival and Bone Loss. Blood Adv (2017) 1(27):2656-66. doi: 10.1182/bloodadvances.2017010801

26. Wang G, Ning FY, Wang JH, Yan HM, Kong HW, Zhang YT, et al. Expression of interleukin-32 in Bone Marrow of Patients With Myeloma and its Prognostic Significance. World J Clin cases (2019) 7(24):4234-44. doi: 10.12998/wjcc.v7.i24.4234

27. Hay RT. SUMO: A History of Modification. Mol Cell (2005) 18(1):1-12. doi: 10.1016/j.molcel.2005.03.012

28. Gill G. SUMO and Ubiquitin in the Nucleus: Different Functions, Similar Mechanisms? Genes Dev (2004) 18(17):2046-59. doi: 10.1101/gad.1214604

29. Rizwani W, Schaal C, Kunigal S, Coppola D, Chellappan S. Mammalian Lysine Histone Demethylase KDM2A Regulates E2F1-mediated Gene Transcription in Breast Cancer Cells. PloS One (2014) 9(7):e100888. doi: 10.1371/journal.pone. 0100888

30. Wagner KW, Alam H, Dhar SS, Giri U, Li N, Wei Y, et al. KDM2A Promotes Lung Tumorigenesis by Epigenetically Enhancing ERK1/2 Signaling. J Clin Invest (2013) 123(12):5231-46. doi: 10.1172/JCI68642

31. Lin $\mathrm{Q}, \mathrm{Wu} \mathrm{Z}$, Yue X, Yu X, Wang Z, Song X, et al. ZHX2 Restricts Hepatocellular Carcinoma by Suppressing Stem Cell-Like Traits Through 
KDM2A-mediated H3K36 Demethylation. EBioMedicine (2020) 53:102676. doi: 10.1016/j.ebiom.2020.102676

32. Okamoto K, Tanaka Y, Tsuneoka M. Sf-KDM2A Binds to Ribosomal RNA Gene Promoter, Reduces H4K20me3 Level, and Elevates Ribosomal RNA Transcription in Breast Cancer Cells. Int J Oncol (2017) 50(4):1372-82. doi: 10.3892/ijo.2017.3908

33. Wang F, Qi XM, Wertz R, Mortensen M, Hagen C, Evns J, et al. P38gamma MAPK is Essential for Aerobic Glycolysis and Pancreatic Tumorigenesis. Cancer Res (2020) 80(16):3251-64. doi: 10.1158/0008-5472.CAN-19-3281

34. Robinson AJ, Hopkins GL, Rastogi N, Hodges M, Doyle M, Davies S, et al. Reactive Oxygen Species Drive Proliferation in Acute Myeloid Leukemia Via the Glycolytic Regulator PFKFB3. Cancer Res (2020) 80(5):937-49. doi: 10.1158/0008-5472.CAN-19-1920

35. Liu J, Liu ZX, Wu QN, Lu YX, Wong CW, Miao L, et al. Long Noncoding RNA AGPG Regulates PFKFB3-mediated Tumor Glycolytic Reprogramming. Nat Commun (2020) 11(1):1507. doi: 10.1038/s41467-020-15112-3

36. Minchenko A, Leshchinsky I, Opentanova I, Sang N, Srinivas V, Armstead V, et al. Hypoxia-Inducible factor-1-mediated Expression of the 6phosphofructo-2-kinase/fructose-2,6-bisphosphatase-3 (PFKFB3) Gene. Its Possible Role in the Warburg Effect. J Biol Chem (2002) 277(8):6183-7. doi: 10.1074/jbc.M110978200

37. Herrero-Mendez A, Almeida A, Fernandez E, Maestre C, Moncada S, Bolanos JP. The Bioenergetic and Antioxidant Status of Neurons is Controlled by Continuous Degradation of a Key Glycolytic Enzyme by APC/C-Cdh1. Nat Cell Biol (2009) 11(6):747-52. doi: 10.1038/ncb1881

38. Marsin AS, Bouzin C, Bertrand L, Hue L. The Stimulation of Glycolysis by Hypoxia in Activated Monocytes is Mediated by AMP-activated Protein
Kinase and Inducible 6-Phosphofructo-2-Kinase. J Biol Chem (2002) 277 (34):30778-83. doi: 10.1074/jbc.M205213200

39. Zhao S, Xu W, Jiang W, Yu W, Lin Y, Zhang T, et al. Regulation of Cellular Metabolism by Protein Lysine Acetylation. Science (2010) 327(5968):1000-4. doi: 10.1126/science.1179689

40. Wade SM, Ohnesorge N, McLoughlin H, Biniecka M, Carter SP, Trenkman M, et al. Dysregulated miR-125a Promotes Angiogenesis Through Enhanced Glycolysis. EBioMedicine (2019) 47:402-13. doi: 10.1016/j.ebiom.2019.08.043

41. Nold-Petry CA, Rudloff I, Baumer Y, Ruvo M, Marasco D, Botti P, et al. Il-32 Promotes Angiogenesis. J Immunol (2014) 192(2):589-602. doi: 10.4049/ jimmunol.1202802

42. Yan H, Dong M, Liu X, Shen Q, He D, Huang X, et al. Multiple Myeloma CellDerived IL-32gamma Increases the Immunosuppressive Function of Macrophages by Promoting Indoleamine 2,3-Dioxygenase (IDO) Expression. Cancer Lett (2019) 446:38-48. doi: 10.1016/j.canlet.2019.01.012

Conflict of Interest: The authors declare that the research was conducted in the absence of any commercial or financial relationships that could be construed as a potential conflict of interest.

Copyright (c) $2021 \mathrm{Liu}, \mathrm{Li}$, Wang, Meng, Wang, Zhao, Xu, Cai and Hu. This is an open-access article distributed under the terms of the Creative Commons Attribution License (CC BY). The use, distribution or reproduction in other forums is permitted, provided the original author(s) and the copyright owner(s) are credited and that the original publication in this journal is cited, in accordance with accepted academic practice. No use, distribution or reproduction is permitted which does not comply with these terms. 